Pacific Journal of Mathematics

作 


\title{
ON A REGULAR SEMIGROUP IN WHICH THE IDEMPOTENTS FORM A BAND
}

\author{
MIYUKI YAMADA
}

This paper is a continuation of a previous paper, in which the structure of certain regular semigroups, called generalized inverse semigroups, has been studied. A semigroup is called strictly regular if it is regular and the set of all its idempotents is a subsemigroup. A generalized inverse semigroup is strictly regular, but the converse is not true. Hence, the class of generalized inverse semigroups is properly contained in the class of strictly regular semigroups. The main purpose of this paper is to establish some results which clarify the structure of strictly regular semigroups. The concept of a quasi-direct product of a band (that is, an idempotent semigroup) and an inverse semigroup is introduced, and in particular it is proved that any semigroup is strictly regular if and only if it is a quasi-direct product of a band and an inverse semigroup.

A regular semigroup $S$ (for the definition, see [1]) is called strictly regular if the set $E$ of idempotents of $S$ is a subsemigroup of $S$. If the set $E$ of a regular semigroup $S$ satisfies a (nontrivial) permutation identity $x_{1} x_{2} \cdots x_{n}=x_{\pi(1)} x_{\pi(2)} \cdots x_{\pi(n)}$, where $\pi$ is a (nontrivial) permutation of $1,2, \cdots, n$, then it can be proved (see [6]) that $E$ is a subsemigroup of $S$ (in fact, $E$ is a normal band ${ }^{1}$ ) and hence $S$ is strictly regular. In this case, $S$ is particularly called a generalized inverse semigroup. Thus any generalized inverse semigroup is strictly regular, but the converse is not true. In the previous paper [6] the author studied the structure of generalized inverse semigroups and established the following theorem:

THEOREM. A semigroup is a generalized inverse semigroup if and only if it is isomorphic to the quasi-direct product of a left normal band, an inverse semigroup and a right normal band.

The main purpose of this paper is to establish a similar result for the class of strictly regular semigroups. Any notation and terminology should be referred to [1], [6], unless otherwise stated.

2. Greatest inverse semigroup decompositions. In this section, we shall determine the greatest inverse semigroup decomposition of a given strictly regular semigroup.

1 An idempotent semigroup $T$ is called a band. If $a b c d=a c b d$ is satisfied for any elements $a, b, c, d$ of $T$, then $T$ is said to be normal. 
Let $R$ be a regular semigroup. Then for any $a \in R$, there exists $x \in R$ such that $a x a=a$ and $x a x=x$. Such an element $x$ is called an inverse of $a$. An inverse of $a$ is not necessarily unique.

Reilly and Scheiblich [4] has proved the following lemma:

Lemma 1. ([4], Lemma 1.3.) Let e be an idempotent of a strictly regular semigroup $S$. Then, every inverse of $e$ is an idempotent.

According to a recent information, the following two lemmas have been also obtained by a paper of T. E. Hall submitted to the Bull. Australian Math. Soc., though the author did not see yet the paper.

LEMMA 2. Let $S$ be a strictly regular semigroup, and $E$ the band (i.e., the idempotent semigroup) consisting of all idempotents of $S$. Let $e, f$ be elements of $E$ such that efe $=e$ and $f e f=f$. Then, for any $a, c \in S^{12}$, any inverse $x$ of aec is also an inverse of afc.

Proof. By the assumption, we have $(a e c) x(a e c)=a e c, x(a e c) x=x$, $e f e=e$ and $f e f=f$. Let $a^{*}, c^{*}$ be any inverses of $a, c$ respectively. (If $a=1$ or $c=1$, then we take 1 as $1^{*}$.) Since $a e c c^{*}(c x a) a^{*} a e c=$ $a e c$, we have $a^{*} a e c c^{*}(c x a) a^{*} a e c c^{*}=a^{*} a e c c^{*}$. Moreover, $c x a\left(a^{*} a e c c^{*}\right) c x a=$ cxaecx $a=c x a$. Since $a^{*} a, c c^{*}$ and $e$ are all idempotents and since $S$ is strictly regular, the element $a^{*} a e c c^{*}$ is an idempotent. Since $a^{*} a e c c^{*}$ is an inverse of $c x a$ and is an idempotent, it follows from Lemma 1 that $c x a$ is also an idempotent. This means that $c x a$ is an inverse of $a^{*} a f c c^{*}$. (In general, let $E \sim \sum\left\{E_{\gamma}: \gamma \in \Gamma\right\}\left(\Gamma\right.$ semilattice; $E_{\gamma}$ rectangular band) be the structure decomposition (for the definition, see [5] or [6]) of $E$. Since efe $=e, f e f=f$, there exists $E_{\gamma}$ such that $e, f \in E_{\gamma}$. Hence for any $\xi \in E_{\alpha}$ and $\eta \in E_{\beta}$, we have $\xi e \eta, \xi f \eta \in E_{\alpha \gamma \beta}$. Therefore any idempotent $\tau$ which is an inverse of $\xi e \eta$ is also an inverse of $\xi f \eta$.)

Hence we have

$$
a^{*} a f c c^{*}(c x a) a^{*} a f c c^{*}=a^{*} a f c c^{*}, a^{*} a f c x a f c c^{*}=a^{*} a f c c^{*}
$$

and accordingly

$$
(a f c) x(a f c)=a f c .
$$

Next, we shall consider about the element $x(a f c) x$.

$$
a f c(x(a f c) x) a f c=a f c(\text { by }(2.1))
$$

and

${ }^{2} S^{1}$ means the adjunction of an identity 1 to $S$ if $S$ has no identity. If $S$ has an identity, then $S^{1}$ means $S$ itself. 


$$
(x(a f c) x) a f c(x(a f c) x)=x(a f c) x \text { (by (2.1)) . }
$$

Therefore, $x(a f c) x$ is an inverse of $a f c$. Accordingly, by using the same method used to get the relation (2.1), we have

$$
(a e c)(x(a f c) x)(a e c)=a e c .
$$

Hence, $x(a e c) x(a f c) x(a e c) x=x(a e c) x$. Since $x$ is an inverse of $a e c$, we have

$$
x(a f c) x=x .
$$

Therefore, it follows from (2.1), (2.3) that $x$ is an inverse of afc.

Let $R$ be a regular semigroup. If a mapping $\varphi: R \rightarrow R$ satisfies the condition

$$
\text { for any } x \in R, x \varphi(x) x=x \text { and } \varphi(x) x \varphi(x)=\varphi(x) \text {, }
$$

then $\varphi$ is called an inverse operator in $R$. It is obvious that $R$ has at least one inverse operator. It is also easy to see that an inverse operator in a regular semigroup $R$ is unique if and only if $R$ is an inverse semigroup.

Now, let $S$ be a regular semigroup. Let $\Omega$ be the set of all inverse operators in $S$. We define a relation $\sigma$ on $S$ as follows:

$$
a \sigma b \text { if and only if }\{\varphi(c a d): \varphi \in \Omega\}=\{\phi(c b d): \varphi \in \Omega\}
$$

for any elements $c, d$ of $S^{1}$. Then, $\sigma$ is clearly an equivalence relation on $S$.

Further, we have

Lemma 3. If $S$ is strictly regular, then $\sigma$ is a congruence relation on $S$.

Proof. Let $a, b$ be elements of $S$ such that $a \sigma b$. Let $h$ be any element of $S$, and $c, d$ any elements of $S^{1}$. Suppose that

$$
x \in\{\varphi(c(a h) d): \varphi \in \Omega\} .
$$

Then, $x \in\{\varphi(c a(h d)): \varphi \in \Omega\}$. Since $a \sigma b$,

$$
x \in\{\varphi(c b(h d)): \varphi \in \Omega\}=\{\varphi(c(b h) d): \varphi \in \Omega\} .
$$

Hence $\{\varphi(c(a h) d): \varphi \in \Omega\} \subset\{\varphi(c(b h) d): \varphi \in \Omega\}$. We can also easily prove the converse relation. Therefore, we have $a h \sigma b h$. By a similar meth-

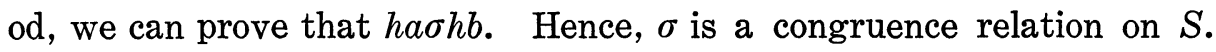


semigroup $S / \sigma$ of $S \bmod \sigma$ is an inverse semigroup. Let $E$ be the band consisting of all idempotents of $S$, and $E \sim \sum\left\{E_{\gamma}: \gamma \in \Gamma\right\}(\Gamma$ semilattice; $E_{\gamma}$ rectangular band) the structure decomposition of $E$.

Then,

(1) for any $e \in E_{\gamma}$, the congruence class $(\in S / \sigma)$ containing $e$ is $E_{r}$, and

(2) the basic semilattice (i.e., the semilattice of idempotents) of $S / \sigma$ is $E / \sigma_{E}=\left\{E_{\gamma}: \gamma \in \Gamma\right\}$, where $\sigma_{E}$ is the restriction of $\sigma$ to $E$.

Proof. It is obvious that $S / \sigma$ is regular. Let $\bar{x}$ denote the congruence class $(\epsilon S / \sigma)$ containing $x$. If $\bar{s} \in S / \sigma$ is an idempotent, then $s \sigma s^{2}$. Hence an inverse $s^{*}$ of $s$ is also an inverse of $s^{2}$, and hence we have $s^{2}=\left(s s^{*} s\right)\left(s s^{*} s\right)=s\left(s^{*} s^{2} s^{*}\right) s=s s^{*} s=s$. Thus, $s$ is an idempotent. It is clear that $\bar{x}$ is an idempotent if $x$ itself is an idempotent. Therefore, it follows that $\bar{x} \in S / \sigma$ is an idempotent if and only if $x$ itself is an idempotent. Next for any $e, f \in E$, we shall show that $e \sigma f$ if and only if $e f e=e$ and $f e f=f$. Suppose at first that $e \sigma f$. Then, $\{\phi(e): \varphi \in \Omega\}=$ $\{\varphi(f): \varphi \in \Omega\}$. Since $e \in\{\varphi(e): \varphi \in \Omega\}$, we have $e \in\{\phi(f): \varphi \in \Omega\}$. Hence $e f e=e$ and $f e f=f$. Conversely, let $e f e=e$ and $f e f=f$. Then, $e \sigma f$ follows from Lemma 2. Thus, eof if and only if efe $=e$ and $f e f=f$. This means that $\sigma$ gives the structure decomposition of $E$ and accordingly that $E / \sigma_{E}$ is isomorphic to $\Gamma$. Since the set $E / \sigma_{E}$ of idempotents of $S / \sigma$ is commutative, $S / \sigma$ is an inverse semigroup having $E / \sigma_{E}$ as its basic semilattice.

Let $G$ be an inverse semigroup, and $L$ the basic semilattice of $G$. Let $S$ be a strictly regular semigroup, and $E$ the band consisting of all idempotents of $S$. If there exists a homomorphism $\xi$ of $S$ onto $G$ such that $\bigcup\left\{\xi^{-1}(t): t \in L\right\}=E$ and the structure decomposition of $E$ is $E \sim \sum\left\{\xi^{-1}(t): t \in L\right\}$, then we say that $S$ is a regular extension of $E$ by $G$.

REMARK. According to Clifford and Preston [2], the above mentioned $\xi$ is unique if it exists. Further, we have the following result: Let $G_{1}, G_{2}$ be inverse semigroups having $L_{1}, L_{2}$ as their basic semilattices respectively. Let $S$ be a strictly regular semigroup, and $E$ the band consisting of all idempotents of $S$. Let $\xi_{1}, \xi_{2}$ be homomorphisms of $S$ onto $G_{1}, G_{2}$ respectively such that $\bigcup\left\{\xi_{1}^{-1}(t): t \in L_{1}\right\}=$ $\bigcup\left\{\xi_{2}^{-1}(u): u \in L_{2}\right\}=E$ and the structure decomposition of $E$ is given as each of $\left\{\xi_{1}^{-1}(t): t \in L_{1}\right\}$ and $\left\{\xi_{2}^{-1}(u): u \in L_{2}\right\}$ (that is, $E \sim \sum\left\{\xi_{1}^{-1}(t): t \in L_{1}\right\}$ and $\left.E \sim \sum\left\{\xi_{2}^{-1}(u): u \in L_{2}\right\}\right)$. Then $G_{1} \cong G_{2}, L_{1} \cong L_{2}$, and $\xi_{1}, \xi_{2}$ induce the same congruence relation on $S$.

THEOREM 1. Let $S$ be a strictly regular semigroup, and $E$ the 
band consisting of all idempotents of $S$. Then, $S$ is a regular extension of $E$ by an inverse semigroup.

Proof. Let $\sigma$ be a congruence relation on $S$ defined by (2.5). Then, it is easy to see from Lemma 4 that $S$ is a regular extension of $E$ by $S / \sigma$.

Now for $\sigma$ defined by (2.5), we have the following theorem:

THEOREM 2. If $S$ is a strictly regular semigroup, then $\sigma$ defined by (2.5) gives the greatest inverse semigroup decomposition of $S$.

Proof. Let $\delta$ be any congruence relation on $S$ such that $S / \delta$ is an inverse semigroup. Let $\widetilde{a}, a \in S$, denote the congruence class containing $a \bmod \delta$. Now, let $x, y$ be elements of $S$ such that $x \delta y$. Since $x \delta y$, any inverse $x^{*}$ of $x$ is also an inverse of $y$. Hence, $\widetilde{x} \widetilde{x}^{*} \widetilde{x}=\widetilde{x}$, $\widetilde{x}^{*} \widetilde{x} \widetilde{x}^{*}=\widetilde{x}^{*}, \widetilde{y} \widetilde{x}^{*} \widetilde{y}=\widetilde{y}$ and $\widetilde{x}^{*} \widetilde{y} \widetilde{x}^{*}=\widetilde{x}^{*}$. Therefore, each of $\widetilde{x}, \widetilde{y}$ is an inverse of $\widetilde{x}^{*}$. By the assumption, $S / \delta$ is an inverse semigroup and hence an inverse of $\widetilde{x}^{*}$ must be unique. Thus we have $\widetilde{x}=\widetilde{y}$, that is, $x \delta y$.

3. Quasi-direct products. In the previous paper [6], the author introduced the concept of quasi-direct products. We shall generalize that concept in this section.

Let $R$ be an inverse semigroup, and $L$ the basic semilattice of $R$. Let $E$ be a band whose structure decomposition is $E \sim \sum\left\{E_{\alpha}: \alpha \in L\right\}$. Define equivalence relations $\pi_{1}, \pi_{2}$ on $E$ as follows:

$$
\begin{aligned}
& e \pi_{1} f \text { if and only if } e f=f \text { and } f e=e . \\
& e \pi_{2} f \text { if and only if } e f=e \text { and } f e=f .
\end{aligned}
$$

For an element $e \in E$, let $\widetilde{e}, \widetilde{e}$ be the equivalence classes containing $e$ $\bmod \pi_{1}, \pi_{2}$ respectively. Put $\widetilde{E}=\{\widetilde{e}: e \in E\}, \widetilde{\widetilde{E}}=\{\widetilde{e}: e \in E\}, \widetilde{E}_{\alpha}=\left\{\widetilde{e}: e \in E_{\alpha}\right\}$ and $\widetilde{\widetilde{E}}_{\alpha}=\left\{\widetilde{e}: e \in E_{\alpha}\right\}, \alpha \in L$. Then, clearly $\widetilde{E}=\sum\left\{\widetilde{E}_{\alpha}: \alpha \in L\right\}$ and $\widetilde{\widetilde{E}}=$ $\sum\left\{\widetilde{\widetilde{E}}_{\alpha}: \alpha \in L\right\}$ (where $\Sigma$ means disjoint sum). Further, for any $e \in E_{\alpha}$, $(\widetilde{e}, \widetilde{e})$ is contained in the product set $\widetilde{E}_{\alpha} \times \widetilde{E}_{\alpha}$ of $\widetilde{E}_{\alpha}$ and $\widetilde{E}_{\alpha}$. Conversely for any $(\widetilde{e}, \widetilde{f}) \in \widetilde{E}_{\alpha} \times \widetilde{\widetilde{E}}_{\alpha}$, there exists a unique element $h$ of $E_{\alpha}$ such that $(\tilde{h}, \tilde{h})=(\tilde{e}, \tilde{f})$. Since $R$ is an inverse semigroup, every element $\xi$ of $R$ has a unique inverse. We shall denote it by $\xi^{-1}$.

To each ordered pair $(\xi, \eta)$ of elements $\xi, \eta$ of $R$, let correspond a mapping $\rho_{(\xi, \eta)}:\left(\widetilde{E}_{\xi \xi-1} \times{\widetilde{E_{\xi}-1 \xi}}_{\xi}\right) \times\left(\widetilde{E}_{\eta \eta-1} \times \widetilde{E}_{\eta-1 \eta}\right) \rightarrow \widetilde{E}_{\xi \eta(\xi \eta)-1} \times \widetilde{E}_{(\xi \eta)-1 \xi \eta}$. If the system $\left\{\rho_{(\xi, \eta)}: \xi, \eta \in R\right\}$ of these mappings $\rho_{(\xi, \eta)}$ satisfies the follow- 
ing condition (3.3), then this system $\left\{\rho_{(\xi, \eta)}: \xi, \eta \in R\right\}$ is called a set of quasi-direct factors of $E$ with respect to $R$ :

Hereafter, we shall use the following notations.

$$
\rho_{(\xi \eta, \nu)}{ }^{\circ} L \rho_{(\xi, \eta)}\left(\left(\widetilde{e_{1}}, \widetilde{f_{1}}\right),\left(\widetilde{e_{2}}, \widetilde{\tilde{f}_{2}}\right),\left(\widetilde{e_{3}}, \widetilde{\tilde{f}_{3}}\right)\right)
$$

means

$$
\rho_{(\xi \eta, \nu)}\left(\rho_{(\xi, \eta)}\left(\left(\widetilde{e_{1}}, \widetilde{\tilde{f}_{1}}\right),\left(\widetilde{e_{2}}, \tilde{\tilde{f}_{2}}\right)\right),\left(\widetilde{e_{3}}, \widetilde{\tilde{f}_{3}}\right)\right),
$$

and

$$
\rho_{(\xi, \eta \nu)}{ }^{\circ} \rho_{(\eta, \nu)}\left(\left({\widetilde{e_{1}}}_{1}, \widetilde{\tilde{f}}_{1}\right),\left(\widetilde{e_{2}}, \widetilde{\tilde{f}}_{2}\right),\left(\widetilde{e}_{3}, \widetilde{\tilde{f}}_{3}\right)\right)
$$

means

$$
\left.\rho_{(\xi, \eta \nu)}\left(\left(\widetilde{e_{1}}, \widetilde{\tilde{f}_{1}}\right), \rho_{(\eta, \nu)}\left(\widetilde{e_{2}}, \widetilde{\tilde{f}_{2}}\right),\left(\widetilde{e_{3}}, \widetilde{\tilde{f}_{3}}\right)\right)\right)
$$

for elements $e_{1}, f_{1}, e_{2}, f_{2}, e_{3}, f_{3}$ such that $e_{1} \in E_{\xi^{-1}}, f_{1} \in E_{\xi^{-1} \xi^{\xi}}, e_{2} \in E_{\eta \eta-1}$, $f_{2} \in E_{\eta-1 \eta}, e_{3} \in E_{\nu \nu-1}$ and $f_{3} \in E_{\nu-1_{\nu}}$.

$$
\begin{aligned}
& \text { (1) If } \xi, \eta \in L \text {, then } \rho_{(\xi, \eta)}\left(\left({\widetilde{e_{1}}}_{1}, \tilde{\tilde{f}}_{1}\right),\left(\widetilde{e_{2}}, \widetilde{\tilde{f}_{2}}\right)\right)=(\widetilde{e f}, \widetilde{e f}) \text {, where } \\
& \text { (2) } \rho_{(\xi \eta, \nu)}{ }^{\circ} L \rho_{(\xi, \eta)}=\rho_{(\xi, \eta \nu)}{ }^{\circ} R \rho_{(\eta, \nu)} \text { for all } \xi, \eta, \nu \in R \text {. } \\
& \text { (3) For any } \xi \in R, e \in E_{\xi \xi^{-1}} \text { and } f \in E_{\xi^{-1} \xi} \text {, there exist } \\
& h \in E_{\xi^{-1} \xi} \text { and } k \in E_{\xi \xi-1} \text { such that } \\
& \rho_{\left(\xi, \xi^{-1 \xi)}\right.}{ }^{\circ} \rho_{\left(\xi^{-1, \xi}\right)}((\widetilde{e}, \tilde{\tilde{f}}),(\tilde{h}, \tilde{\tilde{k}}),(\widetilde{e}, \tilde{\tilde{f}}))=(\widetilde{e}, \tilde{\tilde{f}}) \text {. }
\end{aligned}
$$

The author does not know whether such a system $\left\{\rho_{(\xi, \eta)}: \xi, \eta \in R\right\}$ always exists or not for given $R$ and $E$. However, we shall show later that a set of quasi-direct factors of $E$ with respect to $R$ always exists if $E, R$ have some special types.

Now, suppose that $\left\{\rho_{(\xi, \eta)}: \xi, \eta \in R\right\}$ is a set of quasi-direct factors of $E$ with respect to $R$. Let $E \times R=\left\{((\widetilde{e}, \tilde{f}), \xi): e \in E_{\xi^{-1}-1}, f \in E_{\xi^{-1} \xi}, \xi \in R\right\}$, and define multiplication in $E \times R$ as follows:

$$
\left(\left(\widetilde{e}_{1}, \tilde{\tilde{f}}_{1}\right), \xi\right)\left(\left(\widetilde{e}_{2}, \tilde{f}_{2}\right), \eta\right)=\left(\rho_{(\xi, \eta)}\left(\left(\widetilde{e}_{1}, \tilde{\tilde{f}}_{1}\right),\left(\widetilde{e}_{2}, \tilde{\tilde{f}}_{2}\right)\right), \xi \eta\right) .
$$

Then, $E \times R$ becomes a strictly regular semigroup which has $R$ as its homomorphic image and embeds $E$ as the band of its idempotents. It is easy to see from the definition of the multiplication in $E \times R$ and (1) of (3.3) that $E$ is embedded in $E \times R$ as the band of idempotents of $E \times R$ and $R$ is a homomorphic image of $E \times R$, while it follows from (2), (3) of (3.3) that $E \times R$ is a strictly regular semigroup. Hereafter, we shall call $E \times R$ the quasi-direct product of $E$ and $R$ determined by $\left\{\rho_{(\xi, \eta)}: \xi, \eta \in R\right\}$. 
ExAMPLES. I. Let $R$ be a weakly $C$-inversive semigroup (see [6]; Ljapin [3] has called $R$ a completely regular inverse semigroup), that is a semigroup such that

(1) the idempotents of $R$ form a semilattice $L$,

(2) there exists a subgroup $R(\alpha)$ of $R$ containing $\alpha$ for every $\alpha \in L$, and the collection $\{R(\alpha): \alpha \in L\}$ of all $R(\alpha)$ satisfies (a) $R=$ $\sum\{R(\alpha): \alpha \in L\}$, and (b) $R(\beta) R(\gamma) \subset R(\beta \gamma)$ for all $\beta, \gamma \in L$.

Of course, $R$ is an inverse semigroup and satisfies $\xi \xi^{-1}=\xi^{-1} \xi$ and $(\xi \eta)(\xi \eta)^{-1}=(\xi \eta)^{-1}(\xi \eta)=\xi \xi^{-1} \eta \eta^{-1}$ for all $\xi, \eta \in R$. Let $E$ be a band having $E \sim \sum\left\{E_{\alpha}: \alpha \in L\right\}$ as its structure decomposition. Now, define a mapping $\rho_{(\xi, \eta)}:\left(\widetilde{E}_{\xi \xi^{-1}} \times \widetilde{\widetilde{E}}_{\xi \xi^{-1}}\right) \times\left(\widetilde{E}_{\eta \eta-1} \times \widetilde{\widetilde{E}}_{\eta \eta^{-1}}\right) \rightarrow \widetilde{E}_{\xi \eta(\xi \eta)-1} \times \widetilde{\widetilde{E}}_{(\xi \eta)^{-1} \xi_{\eta}}$ for every ordered pair $(\xi, \eta)$ of elements of $R$ as follows:

$$
\rho_{(\xi, \eta)}\left(\left(\widetilde{e}_{1}, \tilde{\tilde{f}}_{1}\right),\left(\widetilde{e}_{2}, \tilde{\tilde{f}}_{2}\right)\right)\left(=\rho_{(\xi, \eta)}((\widetilde{e}, \widetilde{e}),(\widetilde{f}, \tilde{\tilde{f}}))\right)=(\widetilde{e f}, \widetilde{e f}),
$$

where $e, f$ are elements of $E_{\xi \xi^{-1}}$ and $E_{\eta \eta^{-1}}$ respectively such that $\widetilde{e}=$ $\widetilde{e}_{1}, \widetilde{\tilde{e}}=\tilde{\tilde{f}}_{1}, \tilde{f}=\widetilde{e}_{2}$ and $\tilde{\tilde{f}}=\tilde{\tilde{f}}_{2}$. The existence of such elements $e, f$ and their uniqueness are easily verified.

Then the system $\left\{\rho_{(\xi, \eta)}: \xi, \eta \in R\right\}$ satisfies the conditions (1), (2), (3) of (3.3) and becomes a set of quasi-direct factors of $E$ respect to $R$. Hence, there exists the quasi-direct product $E \times R$ of $E$ and $R$ determined by $\left\{\rho_{(\xi, \eta)}: \xi, \eta \in R\right\}$. That is,

$$
\begin{aligned}
& E \times R=\left\{((\widetilde{e}, \tilde{f}), \xi): e \in E_{\xi^{-1}}, f \in E_{\xi^{-1} \xi}, \xi \in R\right\} \text {, and multi- } \\
& \text { plication in } E \times R \text { is given by }
\end{aligned}
$$

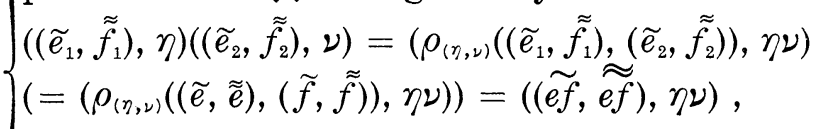

$$
\begin{aligned}
& \text { where } e, f \text { are elements of } E_{\eta \eta^{-1}} \text { and } E_{\nu \nu-1} \text { respectively } \\
& \text { such that } \widetilde{e}=\widetilde{e}_{1}, \widetilde{e}=\widetilde{f}_{1}, \widetilde{f}=\widetilde{e}_{2} \text { and } \tilde{\tilde{f}}=\widetilde{\tilde{f}}_{2} \text {. }
\end{aligned}
$$

On the other hand, let $E \bowtie R(L)$ be the spined product (for the definition of spined products, see [5] or [6]) of $E$ and $R$ with respect to L. Then $E \bowtie R(L)=\sum\left\{E_{\alpha} \times R(\alpha): \alpha \in L\right\}$ by the definition of spined products. Define a mapping $\varphi: E \bowtie R(L) \rightarrow E \times R$ as follows: $\varphi(e, \xi)=$ $((\widetilde{e}, \widetilde{e}), \xi),(e, \xi) \in E \bowtie R(L)$. Then it is easy to see that $\phi$ is an isomorphism of $E \bowtie R(L)$ onto $E \times R$. Hence, in this case the quasidirect product $E \times R$ means the spined product $E \bowtie R(L)$.

II. Let $R$ be an inverse semigroup, and $L$ the basic semilattice of $R$. Let $E$ be a normal band having the structure decomposition $E \sim \sum\left\{E_{\alpha}: \alpha \in L\right\}$. Since $E$ is a normal band, $\widetilde{E}$ and $\widetilde{E}$ are a left normal band and a right normal band respectively (see [6], [7]); hence $\widetilde{e f}=\widetilde{e f}$ for $\tilde{e}, \tilde{f} \in \widetilde{E}$ and $\tilde{e} \tilde{\tilde{f}}=\widetilde{\widetilde{e f}}$ for $\tilde{e}, \tilde{\tilde{f}} \in \widetilde{\widetilde{E}}$. 
Now, define a mapping $\rho_{(\xi, \eta)}:\left(\widetilde{E}_{\xi^{-1}} \times{\widetilde{E_{\xi}-1 \xi}}\right) \times\left(\widetilde{E}_{\eta \eta^{-1}} \times{\widetilde{E_{\eta}-1 \eta}}\right) \rightarrow$ $\widetilde{E}_{\xi \eta(\xi \eta)-1} \times \widetilde{\widetilde{E}}_{(\xi \eta)-1 \xi \eta \eta}$ for every ordered pair $(\xi, \eta)$ of elements $\xi, \eta$ of $R$ as follows:

$$
\rho_{(\xi, \eta)}\left(\left(\widetilde{e_{1}}, \widetilde{\tilde{f}}\right),\left(\widetilde{e_{2}}, \widetilde{\tilde{f}}\right)\right)=\left(\widetilde{e_{1} h}, \widetilde{g f_{2}}\right) \text {, }
$$

where $h, g$ are any elements of $E_{(\xi \eta)(\xi \eta)-1}$ and $E_{(\xi \eta)-1(\xi \eta)}$ respectively.

It was proved by [6] that $\widetilde{e_{1} h}$ and $\widetilde{g f_{2}}$ do not depend on the selection of $h, g$ and hence $\rho_{(\xi, \eta)}$ is well-defined. It is also seen from [6] that the system $\left\{\rho_{(\xi, \eta)}: \xi, \eta \in R\right\}$ satisfies (1), (2), (3) of (3.3) and becomes a set of quasi-direct factors of $E$ with respect to $R$. Hence, we can consider the quasi-direct product $E \times R$ of $E$ and $R$ determined by $\left\{\rho_{(\xi, \eta)}: \xi, \eta \in R\right\}$.

That is,

$$
\begin{aligned}
& E \times R=\left\{((\widetilde{e}, \tilde{\tilde{f}}), \nu): e \in E_{\nu \nu}, f \in E_{\nu-1_{\nu}}, \nu \in R\right\} \text {, and multi- } \\
& \text { plication in } E \times R \text { is defined by } \\
& \left\{\left(\left(\widetilde{e}_{1}, \tilde{\tilde{f}}_{1}\right), \xi\right)\left(\left({\widetilde{e_{2}}}_{2}, \tilde{\tilde{f}}_{2}\right), \eta\right)=\left(\rho_{(\xi, \eta)}\right)\left(\left(\widetilde{e}_{1}, \tilde{\tilde{f}}_{1}\right),\left(\widetilde{e}_{2}, \tilde{\tilde{f}}_{2}\right)\right), \xi \eta\right) \\
& =\left(\left(\widetilde{e_{1} h}, \widetilde{\widetilde{g f}}\right), \xi \eta\right)=\left(\left(\widetilde{e_{1}} \widetilde{h}, \widetilde{g} \tilde{f}_{2}\right), \xi \eta\right) \text {, } \\
& \text { where } h, g \text { are any elements of } E_{\xi \eta(\xi \eta)-1} \text { and } E_{(\xi \eta)^{-1} \xi \eta} \text { re- } \\
& \text { spectively. }
\end{aligned}
$$

On the other hand, we can also consider the quasi-direct product $Q(\widetilde{E} \otimes R \otimes \widetilde{\widetilde{E}} ; L)$ of $\widetilde{E}, \widetilde{\widetilde{E}}$ and $R$ in the sense of [6]. Define a mapping $\varphi: Q(\widetilde{E} \otimes R \otimes \widetilde{\widetilde{E}} ; L) \rightarrow E \times R$ by $\varphi((\widetilde{e}, \xi, \widetilde{\tilde{f}}))=((\widetilde{e}, \widetilde{\tilde{f}}), \xi), e \in E_{\xi \xi-1}, f \in E_{\xi^{-1} \xi}$, $\xi \in R$. Then, it is easy to verify that this $\varphi$ is an isomorphism of $Q(\widetilde{E} \otimes R \otimes \widetilde{E} ; L)$ onto $E \times R .^{3} \quad$ Hence, the concept of quasi-direct products just introduced above is a generalization of the old concept of quasi-direct products introduced by [6].

Now, let $R$ be an inverse semigroup whose basic semilattice is $L$. Let $E$ be a band having $L$ as its structure semilattice (for the definition of structure semilattices, see [6]). Examples I and II show that there exists a quasi-direct product of $E$ and $R$ if, in particular, $R$ is a union of groups or $E$ is a normal band. However, in case that $R$ and $E$ have no restriction we do not know whether there exists a quasi-direct product of $E$ and $R$ or not. Therefore, we state it as an open problem:

${ }^{3}$ Moreover, we have the following result: If $R, E$ are the inverse semigroup and the normal band given in II, then a quasi-direct product of $E$ and $R$ is uniquely determined up to isomorphisms and is isomorphic to $Q(\widetilde{E} \otimes R \otimes \widetilde{\widetilde{E}} ; L)$ (hence of course to the above-mentioned $E \times R$ ). A proof of this result will be given later elsewhere. 
Problem. Let $R$ be an inverse semigroup whose basic semilattice is $L$. Let $E$ be a band having $L$ as its structure semilattice. Is there a quasi-direct product of $E$ and $L$ ? In case that a quasi-direct product of $E$ and $L$ exists, is it unique?

4. A structure theorem. In this section, we shall show that any strictly regular semigroup is isomorphic to a quasi-direct product of a band and an inverse semigroup. More precisely, let $S$ be a strictly regular semigroup and $E$ the band consisting of all idempotents of $S$. Let $\sigma$ be the congruence relation on $S$ which gives the greatest inverse semigroup decomposition of $S$. Then as was shown in Theorem $1, S$ is a regular extension of $E$ by $S / \sigma$. Further it will be shown in this section that such a regular extension of $E$ by $S / \sigma$ which is isomorphic to $S$ can be obtained as a quasi-direct product of $E$ and $S / \sigma$.

Let $S$ be a strictly regular semigroup, and $E$ the band consisting of all idempotents of $S$. Let $E \sim \sum\left\{E_{\alpha}: \alpha \in L\right\}(L$ semilattice) be the structure decomposition of $E$. Let $\sigma$ be the congruence relation on $S$ which gives the greatest inverse semigroup decomposition of $S$. Put $S / \sigma=R$. Let $\bar{x}$ denote the congruence class containing $x \in S \bmod \sigma$. As was shown in the $\S 2, E / \sigma_{E}$ (where $\sigma_{E}$ is the restriction of $\sigma$ to $E$ ), is the basic semilattice of $S / \sigma$. Hence we can assume that $E / \sigma_{E} \equiv L$. Of course, in this case $E / \sigma_{E}=\left\{E_{\alpha}: \alpha \in L\right\}=\left\{E_{\vec{e}}: \bar{e} \in E / \sigma_{E}\right\} .^{4}$

Now, we construct a set of quasi-direct factors $\rho_{(\bar{x}, \bar{y})}$ of $E$ with respect to $R$ as follows: Let $\widetilde{E}=E / \pi_{1}$ and $\widetilde{E}=E / \pi_{2}$, where $\pi_{1}, \pi_{2}$ are the equivalence relations on $E$ defined by (3.1) and (3.2) respectively. Let $\widetilde{E}_{\bar{e}}=E_{\bar{e}} / \pi_{1}$ and ${\widetilde{E_{e}}}_{\bar{e}} / \pi_{2}$. For every ordered pair $(\bar{x}, \bar{y})$ of elements $\bar{x}, \bar{y}$ of $R$, define a mapping

$$
\rho_{(\bar{x}, \bar{y})}:\left(\widetilde{E}_{\bar{x} \bar{x}^{-1}} \times{\widetilde{E_{\bar{x}}-1 \bar{x}}}_{\bar{x}}\right) \times\left(\widetilde{E}_{\bar{y} \bar{y}^{-1}} \times{\widetilde{E_{\bar{y}}-\bar{y}_{\bar{y}}}}\right) \longrightarrow \widetilde{E}_{\overline{x y}(\overline{x y})^{-1}} \times{\widetilde{\widetilde{E}_{(\overline{x y})}-1 \overline{x y}}}
$$

by

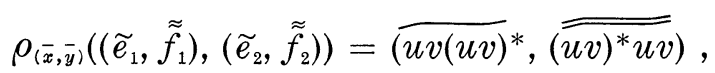

where $u, v$ are elements of $S$ such that $\bar{u}=\bar{x}, \bar{v}=\bar{y}, \widetilde{u u^{*}}=\widetilde{e_{1}}, \widetilde{\widetilde{u^{*} u}}=$ $\widetilde{\tilde{f}_{1}}, \widetilde{v v^{*}}={\widetilde{e_{2}}}_{\text {and }} \widetilde{v^{*} v}=\widetilde{\tilde{f}_{2}}\left(u^{*}, v^{*},(u v)^{*}\right.$ are inverses of $u, v, u v$ respectively ${ }^{5}$ ). For an element $x$ of a regular semigroup, hereafter we shall use the notation $x^{*}$ to denote an inverse of $x$. Hence, for example, $a^{*}$ means any inverse of $a$.

The existence of $u, v$ in (4.1) and their uniqueness are obvious

${ }^{4}$ When we regard $\bar{e}$ as a subset of $E$, we denote it by $E_{\bar{e}}$. Hence, $E_{\bar{e}}=E_{\alpha}$ if and only if $\bar{e} \equiv \alpha$, i.e., $E_{\alpha} \ni e$.

5 For any two inverses $u_{1}, u_{2}$ of $u, \widetilde{u u_{1}}=\widetilde{u u_{2}}$ and $\underset{u_{1} u}{\approx}=\widetilde{u_{2} u}$. Hence, $\widetilde{u u^{*}}$ and $\widetilde{u^{*} u}$ do not depend on the selection of an inverse $u^{*}$ of $u$. 
from the following result:

Lemma 5. For any elements $\bar{x}$ of $R$, e of $E_{\bar{x} \bar{x}-1}$ and $f$ of $E_{\bar{x}-1 \bar{x}}$, there exists a unique element $u$ of $S$ such that $\bar{u}=\bar{x}, \widetilde{u u^{*}}=\widetilde{e}$ and $\overline{\overline{u^{*} u}}=\tilde{\tilde{f}} . \quad$ In fact, $u=$ exf has these properties.

Proof. Let $u=e x f$. Since $\bar{x}=\overline{x x^{*}} \bar{x} \overline{x^{*} x}=\left(\bar{x} \bar{x}^{-1}\right) \bar{x}\left(\bar{x}^{-1} \bar{x}\right)=\bar{e} \bar{x} \bar{f}=$ $\overline{e f x}$, we have $\bar{x}=\overline{e x f}=\bar{u}$. Now, we can take $f x^{*} e$ as an inverse of $u$ (see [4]). Hence, let $u^{*}=f x^{*} e$. Since $\bar{e}=\bar{x} \bar{x}^{-1}=\bar{x} \overline{x^{*}}=\bar{u} \overline{u^{*}}=\overline{u u^{*}}$, both $e$ and $u u^{*}$ are contained in $E_{\bar{e}}$. Hence, $e=e u u^{*} e=e(e x f)\left(f x^{*} e\right) e=$ $(e x f)\left(f x^{*} e\right)=u u^{*}$. That is, $e=u u^{*}$. Similarly, we obtain $u^{*} u=f$. Therefore, of course $\widetilde{e}=\widetilde{u u^{*}}$ and $\tilde{\tilde{f}}=\widetilde{\widetilde{u^{*} u}}$. Next, we shall prove that such an element $u$ is unique. Let $v$ be any element of $S$ such that $\bar{v}=\bar{x}, \overline{v v^{*}}=\widetilde{e}$ and $\overline{\overline{v^{*} v}}=\tilde{\tilde{f}}$. Since $\widetilde{u u^{*}}=\widetilde{v v^{*}}, \overline{\overline{u^{*} u}}=\overline{\overline{v^{*} v}}$ and $\bar{u}=\bar{v}$, we have $v v^{*} u u^{*}=u u^{*}, u^{*} u v^{*} v=u^{*} u$ and $u \sigma v$. Since $u \sigma v, v^{*} u v^{*}=v^{*}$. Hence,

$$
\begin{aligned}
u & =u u^{*} u=\left(v v^{*} u u^{*}\right) u=v v^{*} u\left(u^{*} u\right)=v v^{*} u\left(u^{*} u v^{*} v\right) \\
& =v v^{*}\left(u u^{*} u\right) v^{*} v=v\left(v^{*} u v^{*}\right) v=v v^{*} v=v .
\end{aligned}
$$

Consequently, $u=v$.

When we consider an element $\bar{x}$ of $R$ as a subset of $S$, we shall denote it by $S_{\bar{x}}$. Of course $S_{\bar{x}}=S_{\bar{y}}$ if and only if $\bar{x}=\bar{y}$, i.e., $x \sigma y$.

Lemma 6. For $\bar{x} \in R$,

(1) $S_{\bar{x}}=\left\{e x f: e \in S_{\bar{x}_{\bar{x}}^{-1}}\left(=E_{\bar{x} \bar{x}-1}\right), f \in S_{\bar{x}^{-1} \bar{x}}\left(=E_{\bar{x}-1 \bar{x}}\right)\right\}$,

(2) $\left|S_{\bar{x}}\right|=\left|\widetilde{E}_{\bar{x} \bar{x}-1}\right|\left|\widetilde{\widetilde{E}}_{\bar{x}-1 \bar{x}}\right|^{6}$, and

(3) for $e, e^{\prime} \in E_{\bar{x} \bar{x}-1}$ and for $f, f^{\prime} \in E_{\bar{x}-1_{\bar{x}}}, e x f=e^{\prime} x f^{\prime}$ if and only if $\widetilde{e}=\widetilde{e}^{\prime}$ and $\widetilde{f}=\widetilde{f^{\prime}}$.

Proof. Let $e x f$ be an element of $\left\{e x f ; e \in S_{\bar{x} \bar{x}-1}, f \in S_{\bar{x}^{-1}-1}\right\}$. Then since $\overline{e x f}=\bar{x} \bar{x}^{-1} \bar{x} \bar{x}^{-1} \bar{x}=\bar{x}$, exf is an element of $S_{\bar{x}}$. Conversely let $y \in S_{\bar{x}}$, and put $y y^{*}=e^{\prime}$ and $y^{*} y=f^{\prime} . \quad \bar{y}=\bar{x}$ implies $y^{*}$ is an inverse of $x$. Hence $y=y y^{*} y=y y^{*} x y^{*} y=e^{\prime} x f^{\prime}$. Therefore, $y$ is contained in the set $\left\{e x f: e \in E_{\bar{x} \bar{x}-1}, f \in E_{\bar{x}-1 \bar{x}}\right\}$. Thus (1) is satisfied. Since (2) is obvious from (1) and (3), we next prove only the part (3). Suppose that exf $=e^{\prime} x f^{\prime}, e, e^{\prime} \in E_{\bar{x} \bar{x}-1}$ and $f, f^{\prime} \in E_{\bar{x}-1 \bar{x}}$. Then $\overline{e x f}=\overline{e^{\prime} x f^{\prime}}=\bar{x}$. As is seen from Lemma 5 , these elements satisfy $\overline{(\operatorname{exf})(\operatorname{exf})^{*}}=\widetilde{e}$, $\left.\widetilde{\left(e^{\prime} x f^{\prime}\right)\left(e^{\prime} x f^{\prime}\right)^{*}}=\widetilde{{\widetilde{e^{\prime}}}^{\prime}}, \overline{(e x f)^{*}(e x f}\right)=\tilde{\tilde{f}}$ and $\overline{\left(\overline{\left.e^{\prime} x f^{\prime}\right)^{*}\left(e^{\prime} x f^{\prime}\right)}\right.}=\widetilde{\tilde{f}^{\prime}}$. Since exf $=$ $e^{\prime} x f^{\prime}$, it follows from the above that $\widetilde{e}=\widetilde{e^{\prime}}$ and $\tilde{\tilde{f}}=\widetilde{\tilde{f}^{\prime}}$. Conversely,

${ }^{6}$ If $A$ is a set, the notation $|A|$ means the cardinality of $A$. 
suppose that $\tilde{e}=\widetilde{\tilde{e}^{\prime}}, \tilde{\tilde{f}}=\tilde{f^{\prime}}, e, e^{\prime} \in E_{\bar{x} \bar{x}^{-1}}$ and $f, f^{\prime} \in E_{\bar{x}^{-1} \bar{x}}$. Then, we have $\left.\overline{e x f^{\prime}}=\bar{x}=\overline{e^{\prime} x f^{\prime}}, \overline{(e x f)(e x f)^{*}}=\widetilde{e}=\widetilde{e^{\prime}}=\overline{\left(e^{\prime} x f^{\prime}\right)\left(e^{\prime} x f^{\prime}\right.}\right)^{*}$ and

$$
\overline{(e x f)^{*}(e x f)}=\tilde{\tilde{f}}=\tilde{\tilde{f}^{\prime}}=\widetilde{\left(e^{\prime} x f^{\prime}\right)^{*}\left(e^{\prime} x f^{\prime}\right)} \text {. }
$$

Hence by Lemma 5, two elements $e x f, e^{\prime} x f^{\prime}$ must be the same.

Corollary. If $R$ is finite, then $|S|=\sum_{\bar{x} \in R}\left|\widetilde{E}_{\bar{x} \bar{x}-1}\right|\left|{\widetilde{E_{\bar{x}}-1 \bar{x}}}_{\mid}\right|$.

Proof. Obvious.

For every ordered pair $(\bar{x}, \bar{y})$ of elements $\bar{x}, \bar{y}$ of $R$, anyway $\rho_{(\bar{x}, \bar{y})}$ is well-defined. Let $\Omega=\left\{\rho_{(\bar{x}, \bar{y})}: \bar{x}, \bar{y} \in R\right\}$ be the collection of all these $\rho_{(\bar{x}, \bar{y})}$. Then, it is easy to see that $Q$ becomes a set of quasi-direct factors of $E$ with respect to $R$, that is, $\Omega$ satisfies the conditions (1), (2), (3) of (3.3). We shall give a proof only for the condition (2) which is the most complicated condition among the three.

We should prove

$$
\begin{aligned}
& \text { (2) of }(3.3): \rho_{(\overline{x y}, \bar{z})}\left(\rho_{(\bar{x}, \bar{y})}\left(\left(\widetilde{e_{1}}, \tilde{\tilde{f}}_{1}\right),\left(\widetilde{e_{2}}, \tilde{\tilde{f}}_{2}\right)\right),\left(\widetilde{e_{3}}, \tilde{\tilde{f}}_{3}\right)\right) \\
& =\rho_{(\bar{x}, \overline{y z})}\left(\left(\widetilde{e}_{1}, \widetilde{\tilde{f}}_{1}\right), \rho_{(\bar{y}, \bar{z})}\left(\left(\widetilde{e}_{2}, \tilde{\tilde{f}}_{2}\right),\left(\widetilde{e}_{3}, \widetilde{\tilde{f}}_{3}\right)\right) .\right.
\end{aligned}
$$

By Lemma 5, there exist unique $u, v, w$ such that $\bar{u}=\bar{x}, \widetilde{u u^{*}}=\widetilde{e}_{1}$, $\widetilde{\widetilde{u^{*} u}}=\tilde{\tilde{f}}, \bar{v}=\bar{y}, \widetilde{v v^{*}}=\widetilde{e}_{2}, \overline{\widetilde{v^{*} v}}=\tilde{\tilde{f}}, \bar{w}=\bar{z}, \widetilde{w w^{*}}=\widetilde{e}_{3}$ and $\overparen{w^{*} w}=\tilde{\tilde{f}}_{3}$. Hence $\rho_{(\bar{x}, \bar{y})}\left(\left(\widetilde{e}_{1}, \widetilde{\tilde{f}}_{1}\right),\left(\widetilde{e}_{2}, \tilde{\tilde{f}}_{2}\right)\right)=\widetilde{\left(u v(u v)^{*}\right.}, \widetilde{\overline{\left.(u v)^{*} u v\right)}}$, and hence

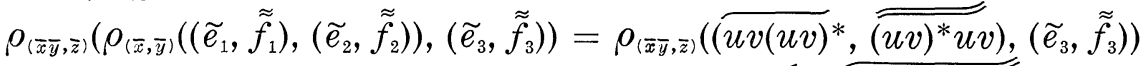

$$
\begin{aligned}
& =\widetilde{\left(u v w(u v w)^{*}\right.}, \widetilde{\left.(u v w)^{*} u v w\right)} \text {. }
\end{aligned}
$$

On the other hand, $\rho_{(\bar{y}, \bar{z})}\left(\left(\widetilde{e}_{2}, \tilde{\tilde{f}_{2}}\right),\left(\widetilde{e_{3}}, \tilde{\tilde{f}_{3}}\right)\right)=\left(\widetilde{\left(v w(v w)^{*}\right.}, \widetilde{(v w)^{*} v w}\right)$. Hence $\rho_{(\bar{x}, \bar{y} \bar{z})}\left(\left(\widetilde{e}_{1}, \widetilde{\tilde{f}}_{1}\right), \rho_{(\bar{y}, \bar{z})}\left(\left({\widetilde{e_{2}}}_{2}, \tilde{\tilde{f}}_{2}\right),\left(\widetilde{e}_{3}, \tilde{\tilde{f}}_{3}\right)\right)\right)=\rho_{(\bar{x}, \bar{y} \bar{z})}\left(\left({\widetilde{e_{1}}}_{1}, \tilde{\tilde{f}}_{1}\right),\left(\widetilde{\left(v w(v w)^{*}\right.}, \widetilde{(v w)^{*} v w}\right)\right)=$ $\widetilde{\left(u v w(u v w)^{*}\right.}, \widetilde{\left.(u v w)^{*} u v w\right)}$. Accordingly, (2) of (3.3) is satisfied. Since $\Omega$ is a set of quasi-direct factors of $E$ with respect to $R$, we can consider the quasi-direct product $E \times R$ of $E$ and $R$ determined by $\Omega$.

Now,

$$
E \times R=\left\{((\widetilde{e}, \tilde{\tilde{f}}), \bar{x}): \bar{x} \in R, e \in E_{\bar{x} \bar{x}-1}, f \in E_{\bar{x}-1_{\bar{x}}}\right\}
$$

and multiplication in $E \times R$ is of course given by

$$
\left.\left(\left(\widetilde{e}_{1}, \widetilde{\tilde{f}}_{1}\right), \bar{x}\right)\left(\left(\widetilde{e}_{2}, \widetilde{\tilde{f}}_{2}\right), \bar{y}\right)=\left(\rho_{(\bar{x}, \bar{y})}\left(\left(\widetilde{e}_{1}, \tilde{\tilde{f}}_{1}\right)\right),\left(\widetilde{e}_{2}, \tilde{\tilde{f}}_{2}\right)\right), \bar{x} \bar{y}\right) \text {. }
$$

As to the connection between these $S$ and $E \times R$, we have the following theorem which is the main result of this paper:

THEOREM 3. Let $S$ be a strictly regular semigroup, and $E$ the band consisting of all idempotents of $S$. Let $R$ be the greatest inverse 
semigroup homomorphic image of $S$. Then, $S$ is isomorphic to a quasi-direct product of $E$ and $R$.

Proof. Take the quasi-direct product $E \times R$ obtained by (4.2), and consider the mapping $\varphi: S \rightarrow E \times R$ defined by $\left.\varphi(x)=\left(\overline{\left(x x^{*}\right.}, \widetilde{\widetilde{x^{*} x}}\right), \bar{x}\right), x \in S$. It is obvious from Lemmas 5 and 6 that $\varphi$ is one-to-one and onto. Further, we have

$$
\begin{aligned}
\varphi(x) \varphi(y) & \left.=\left(\widetilde{\left(x x^{*}\right.}, \widetilde{x^{*} x}\right), \bar{x}\right)\left(\widetilde{\left(y y^{*}, \widetilde{y^{*} y}\right.}, \bar{y}\right) \\
& =\left(\rho_{(\bar{x}, \bar{y})}\left(\widetilde{\left(x x^{*}\right.}, \widetilde{\left.x^{*} x\right)}, \widetilde{\left(y y^{*}, \bar{y}^{*} y\right)}\right), \bar{x} \bar{y}\right)\left(\left(\widetilde{(x y)(x y)^{*}}, \widetilde{\left(\overline{x y)^{*}(x y}\right)}\right), \overline{x y}\right) \\
& =\varphi(x y) .
\end{aligned}
$$

Hence, $\varphi$ is an isomorphism.

Finally the author is indebted to Professor Naoki Kimura for valuable discussions and for substantial help in the development of the material of this paper.

\section{REFERENCES}

1. A. H. Clifford and G. B. Preston, Algebraic theory of semigroups, Vol. I, Amer. Math. Soc., Providence, Rhode Island, 1961.

2. - Algebraic theory of semigroups, Vol. II, Amer. Math. Soc., Providence, Rhode Island, 1967.

3. E. S. Ljapin, Semigroups, Amer. Math. Soc., Providence, Rhode Island, 1963.

4. N. R. Reilly and H. E. Scheiblich, Congruences on regular semigroups, Pacific J. Math. 23 (1967), 349-360.

5. M. Yamada, Strictly inversive semigroups, Science Reports of Shimane University 13 (1964), 128-138.

6. - Regular semigroups whose idempotents satisfy permutation identities, Pacific J. Math. 21 (1967), 371-392.

7. M. Yamada and N. Kimura, Note on idempotent semigroups, II, Proc. Japan Acad. 34 (1958), 110-112.

Received May 12, 1969. This research has been partially supported by the National Science Foundation under Grant GP-7429.

University of Arkansas, Fayetteville, Arkansas and

Shimane University, Matsue, Japan 


\section{PACIFIC JOURNAL OF MATHEMATICS}

\section{EDITORS}

H. SAMELSON

Stanford University

Stanford, California 94305

\section{RichaRd PIERCe}

University of Washington

Seattle, Washington 98105
J. DUGUNDJI

Department of Mathematics

University of Southern California

Los Angeles, California 90007

RICHARD ARENS

University of California

Los Angeles, California 90024

\section{ASSOCIATE EDITORS}

E. F. BECKENBACH

B. H. NeUManN

F. WOLF

K. YosHIDA

\section{SUPPORTING INSTITUTIONS}

UNIVERSITY OF BRITISH COLUMBIA CALIFORNIA INSTITUTE OF TECHNOLOGY UNIVERSITY OF CALIFORNIA MONTANA STATE UNIVERSITY

UNIVERSITY OF NEVADA

NEW MEXICO STATE UNIVERSITY

OREGON STATE UNIVERSITY

UNIVERSITY OF OREGON

OSAKA UNIVERSITY

UNIVERSITY OF SOUTHERN CALIFORNIA
STANFORD UNIVERSITY

UNIVERSITY OF TOKYO

UNIVERSITY OF UTAH

WASHINGTON STATE UNIVERSITY

UNIVERSITY OF WASHINGTON

${ }^{*} \quad{ }^{*} \quad{ }^{*}$
AMERICAN MATHEMATICAL SOCIETY
CHEVRON RESEARCH CORPORATION
TRW SYSTEMS
NAVAL WEAPONS CENTER




\section{Pacific Journal of Mathematics}

\section{Vol. 33, No. $1 \quad$ March, 1970}

Mir Maswood Ali, On some extremal simplexes ................... 1

Silvio Aurora, On normed rings with monotone multiplication........... 15

Silvio Aurora, Normed fields which extend normed rings of integers....... 21

John Kelly Beem, Indefinite Minkowski spaces..................... 29

T. F. Bridgland, Trajectory integrals of set valued functions ........... 43

Robert Jay Buck, A generalized Hausdorff dimension for functions and sets ......................................... 69

Vlastimil B. Dlab, A characterization of perfect rings . . . . . . . . . . . . 79

Edward Richard Fadell, Some examples in fixed point theory ............ 89

Michael Benton Freeman, Tangential Cauchy-Riemann equations and uniform approximation ............................. 101

Barry J. Gardner, Torsion classes and pure subgroups ................ 109

Vinod B. Goyal, Bounds for the solution of a certain class of nonlinear

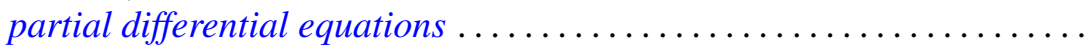

Fu Cheng Hsiang, On C, 1 summability factors of Fourier series at a given

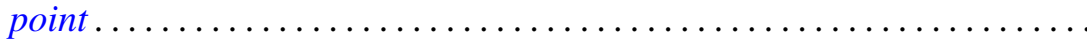

Lawrence Stanislaus Husch, Jr., Homotopy groups of PL-embedding

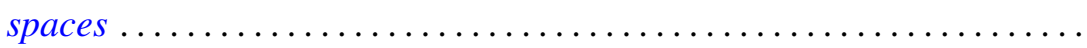

Daniel Ralph Lewis, Integration with respect to vector measures..........

Marion-Josephine Lim, $\mathscr{L}-2$ subspaces of Grassmann product spaces

Stephen J. Pierce, Orthogonal groups of positive definite multilinear functionals

W. J. Pugh and S. M. Shah, On the growth of entire functions of bounded index.

Siddani Bhaskara Rao and Ayyagari Ramachandra Rao, Existence of triconnected graphs with prescribed degrees . . .

Ralph Tyrrell Rockafellar, On the maximal monotonicity of subdifferential

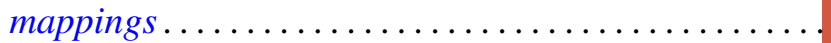

R. Shantaram, Convergence of a sequence of transformations of distribution functions. II ...............................

Julianne Souchek, Rings of analytic functions..............

Ted Joe Suffridge, The principle of subordination applied to functions of several variables...

Wei-lung Ting, On secondary characteristic classes in cobordism

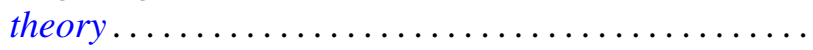

Pak-Ken Wong, Continuous complementors on $B^{*}$-algebras ...

Miyuki Yamada, On a regular semigroup in which the idempotents form a band. 